\title{
An Analysis of Main Affecting Factors on Chinese GDP Growth and their Influence Mechanism
}

\author{
Yufeng Huang \\ Sociology department \\ Peking University \\ Beijing, China \\ Yufengh2013@163.com
}

\author{
Shaojing Shen \\ Guanghua School of Management \\ Peking University \\ Beijing, China \\ shenshaojing1226@163.com
}

\begin{abstract}
In order to understand the main problems of Chinese GDP Growth, the methods of factor analysis, multiple regressions and hierarchical clustering are used to analyze the influences of consumption, investment, export and import on GDP growth, based on the economic data of 31 provinces from 1998 to 2013. The results showed that consumption was the biggest contributor to GDP growth, followed by investment. Export had less effect on GDP growth but was still an unneglectable factor, while import was not really important to GDP. Furthermore, the graded quantitative relationship between GDP growth and typical configurations of consumption, investment and export were found, which was used to assess the potential grades of GDP growth for 31 provinces in China according to the economic data in 2013. It was pointed out that Chinese economy has entered into a GDP low-speed growth stage as a whole. Based on the research results, strengthening consumption and maintaining a suitable investment scale were regarded as an important guarantee of the sustainable and stable development of Chinese economy.
\end{abstract}

Keywords-Gross domestic product; Consumption; Investment; Export; Multiple regressions; Hierarchical clustering

\section{INTRODUCTION}

Gross domestic product (GDP) is a main indicator adopted to measure social and economic development in a region. China's GDP growth for over 20 consecutive years and its total output of present economic gross have captivated the world attention. However in recent 2 years, China's GDP slowdown expectation has revealed that Chinese economy will enter into an important adjustment stage [1]. Under such a background, it is of vital importance in deeply recognizing innate impact mechanism about GDP growth and economic development problems in China. Over the past years, China's economic circle has been adopting investment, consumption and export as the frequent indicator framework [2] in analyzing and judging economic growth; such analytic results are still worth concerning.

In fact, quite a few in-depth analysis and reports are discovered for the impact mechanism of GDP growth based on the analysis of above-mentioned factors and some controversy remains. A few scholars think that Chinese economy has an "export-oriented" development trend and predict that import \& export development and world trade prosperity will become the major momentum of China's economic growth [3]; quite a few scholars insist that although
China's consumption impact is deepened year by year and takes up about $50 \%$ of GDP in recent years, investment is still the most important factor $[4,5]$ in promoting China's economic growth. In view of previous related researches, we have discovered shortcomings, or using fewer sample number or based on single data source in related researches; therefore, much results are hard to convince people. Based on the data of economic indicators such as GDP for 16 years in various provinces (cities), this article makes an in-depth analysis by using several statistic methods, aiming to further specify the relationships between the changes of main important economic factors and China's GDP growth, and to make an objective evaluation of existing potential of China's GDP growth in order to provide favorable basis for China's economic development and structural adjustment.

\section{DATA AND METHODS}

\section{A. Data and initial treatment}

The data of GDP, fixed investment, total retail sales of consumer goods, gross export and total import of 31 provinces (cities) from 1998 to 2013 are adopted-most data come from the annual statistical data published by provincial statistical bureaus in China while a small amount of data come from the Statistical Database of China Social and Economic Development specified in http://www.cnki.net/. Upon initial analysis, the above-mentioned 5 economic indicators of various provinces have shown steady growth trends over the past 16 years; for example, China's annual GDP has increased by nearly 7 times and other indicators have similar changes. So, the original data of sample years suffer from poor comparability and are not able to be used to statistically analyze. In view of rather small difference of economic status between 2 consecutive years for each province, this article transfers an economic indicator $X_{\mathrm{i}}$ of specific year into the increase/decrease change rate $x_{\mathrm{i}}$ with contrasting the value of its previous year: $x_{\mathrm{i}}=\left(X_{\mathrm{i}}-X_{\mathrm{i}-1}\right) / X_{\mathrm{i}-1}$ $\times 100$. Here, $X_{\mathrm{i}}$ and $X_{\mathrm{i}-1}$ refer to the absolute value of original variable of year $i$ and year $i-1 ; x_{\mathrm{i}}$ refers to the relative value transformed. Upon the initial treatment, the number of data samples is reduced from 16 years to 15 years; however, the fluctuation changes among variable samples remain stable which upgrade the comparability and representative property of sample data. 


\section{B. Methods}

Firstly, factor analysis method[6] is adopted to make analysis on the annual changes of GDP field, fixed investment (I) field, total retail sales field of consumer goods (C) , gross export (E) field and total import ( $I m$ ) field, finding out several public factors for each field, which are able to explain above $80 \%$ of total variance of respective variable field. Secondly, correlation analysis method is adopted to make analysis on the relationships between public factors from different economic indicators. On such a basis, multi-linear regression method is used to make analysis on the quantitative relationships between GDP main public factors and other economic indicator field's public factors, and to demonstrate the impact degree of various factors (indicators) on GDP changes. Then the above-mentioned important public factors greatly affecting GDP are used for specified indicators, with which hierarchical clustering method ${ }^{[6]}$ is able to divide and obtain a few specific year types of sample cases ( being helpful for making clear what configuration of I, C, E or Im bring benefits or disadvantages to GDP growth). Ultimately, we attempts to evaluate the present GDP growth potential levels of various provinces (cities) based on the actual values of I, C and E and the similarity analysis with obtained typical configuration year types.

\section{RESULTS AND INITIAL ANALYSIS}

\section{A. Public factors of important economic indicator fields}

The factor analysis on GDP field obtains 3 public factors (the table of analyzed results being omitted). They are able to explain $6.1 \%-51.7 .3 \%$ of variance of original variable GDP field; explain an accumulative degree of $88.5 \%$ of its total variance.

The factor analysis on $I$ field of fixed investment obtains 6 public factors - they are able to interpret $5.7 \%--37.2 \%$ of variance of original variable field and make an accumulative interpretation with $88.7 \%$ of its total variance.

The factor analysis on $\mathrm{C}$ field of total retail sales of consumer goods obtains 4 public factors-they are able to explain the variance of $17.2 \%-26.5 \%$ original variable field and do an accumulative interpretation of $86.1 \%$ of total variance. E field of gross export is expressed by 6 public factors - they are able to interpret $6.9 \%-33.8 \%$ of total variance of original variable field and make an accumulative interpretation of $88.7 \%$ of total variance. Im field of total import is indicated by 9 public factors-they are able to explain $5.3 \%-24.9 \%$ of total variance of original variable and make an accumulative interpretation of $93.3 \%$ of total variance.

Above all, factor analysis method obtains 3 public factors in GDP field and 25 public factors in other 4 economic indicators' variable fields. And the public factors from the same economic indicator field are independent of each other.

\section{B. Correlation between GDP and other indicator variable field's public factors}

Table 1 shows the analytic results in detail. GDP field only includes first 2 public factors which have significant correlation with 4 economic indicator fields' several public factors $\left(I_{1}, I_{3}, C_{1}, C_{2}, C_{3}, E_{1}, E_{4}, I_{1}, I_{3}\right.$ and $\left.I_{4}\right)$. However, the 2 public factors are able to explain $82.4 \%$ of total variance of original variable GDP field.

We draw the following initial conclusion according to the results in Table 1: compared with other 3 economic indicators, the investment $I$ has the greatest impact on GDP and consumption $C$ does No. 2 impact on GDP; and export $E$ is No. 3 impact factor on GDP while import Im is No. 4 impact factor.

\section{Influence of important impact factors on GDP public factors}

In view of some significant correlation between public factors of 4 economic indicators excluding GDP (for example, negative correlation between import $\mathrm{Im}_{1}$ and consumption $C_{1}$ ), the relationships between associated public factors from GDP and from investment, consumption, export are able to explain variable Im's main impact on GDP to a certain extent. Therefore, the influence of total import (Im) on GDP is no longer considered in the following analysis.

- Influence of important factors on No. 1 public factor of GDP

Based on the results of correlation analysis, the investment $I_{1}, I_{3}$, consumption $C_{1}, C_{2}$ and $C_{3}$, export $E_{4}$ are used to make regression analysis with GDP's first public factor $G_{1}$. The regression formula of standardized variables is as follows:

$$
\begin{aligned}
G_{1}= & 2.067 e^{-7}+0.545 C_{3}+0.388 I_{3}+0.352 C_{2} \\
& +0.310 I_{1}+0.264 C_{1}-0.126 E_{4}
\end{aligned}
$$

The fitting degree of this equation is $\mathrm{R}^{2}=0.855$; upon adjustment, $\mathrm{R}^{2}=0.746, \mathrm{~F}=7.846$ (significance level $\mathrm{P}=0.005$ ); the average annual standardized error of the equation is 0.336 ; the standardized fitting error is 0.079 . These parameters and Fig. 1 reveal favorable regression effect as a whole. According to the standardized regression coefficients, consumption has the greatest impact on $G_{1}$; investment has No. 2 impact and export has the least contributions. 
TABLE I. THE CORRELATION COEFFICIENTS BETWEEN THE PUBLIC FACTORS FROM GDP FIELD AND 4 ECONOMIC INDICATOR FIELDS

\begin{tabular}{|c|c|c|c|c|c|c|c|c|c|c|c|c|c|}
\hline & $I_{1}$ & $I_{2}$ & $I_{3}$ & $I_{4}$ & $I_{5}$ & $I_{6}$ & $C_{1}$ & $C_{2}$ & $C_{3}$ & $C_{4}$ & $E_{1}$ & $E_{2}$ & $E_{3}$ \\
\hline$G_{1}$ & $0.619^{* *}$ & 0.083 & $0.444^{*}$ & -0.106 & -0.344 & 0.277 & $0.486^{*}$ & $0.540^{* *}$ & $0.449^{*}$ & 0.409 & -0.202 & 0.345 & 0.325 \\
\hline$G_{2}$ & 0.013 & 0.414 & $-0.578^{* *}$ & -0.219 & 0.060 & 0.391 & -0.407 & 0.109 & 0.372 & -0.071 & $0.918^{* * *}$ & -0.086 & 0.128 \\
\hline \multirow[t]{2}{*}{$G_{3}$} & -0.357 & -0.198 & 0.184 & -0.169 & 0.233 & -0.182 & 0.150 & -0.374 & -0.026 & 0.091 & -0.012 & 0.280 & 0.274 \\
\hline & $E_{4}$ & $E_{5}$ & $E_{6}$ & $I m_{1}$ & $\mathrm{Im}_{2}$ & $\mathrm{Im}_{3}$ & $\mathrm{Im}_{4}$ & $\mathrm{Im}_{5}$ & $\mathrm{Im}_{6}$ & $\mathrm{Im}_{7}$ & $\mathrm{Im}_{8}$ & $\mathrm{Im}_{9}$ & \\
\hline$G_{1}$ & $0.575^{* *}$ & -0.021 & 0.240 & -0.200 & 0.011 & 0.034 & $0.569^{* *}$ & 0.201 & -0.115 & 0.276 & $0.547^{* *}$ & 0.173 & \\
\hline$G_{2}$ & 0.180 & 0.048 & 0.033 & $0.542^{* *}$ & 0.102 & $0.533^{* *}$ & -0.122 & 0.135 & 0.238 & 0.386 & 0.026 & 0.223 & \\
\hline$G_{3}$ & -0.250 & -0.237 & 0.416 & -0.093 & 0.384 & 0.044 & 0.379 & 0.248 & 0.162 & 0.143 & -0.305 & 0.098 & \\
\hline
\end{tabular}

Remark: *** means significance level $\mathrm{P}=0.001 ; * *$ means $\mathrm{P}=0.05$; $*$ means $\mathrm{P}=0.10$.

In view of more independent variables existing in equation 1 , only its first 5 independent variables are selected to make a better regression analysis with $G_{1}$. The standardized regression formula is gained as follows:

$$
\begin{aligned}
G_{1}= & 1.976 e^{-7}+0.493 C_{3}+0.407 I_{3}+0.296 I_{1} \\
& +0.268 C_{2}+0.247 C_{1}
\end{aligned}
$$

Under the condition of reducing 1 independent variable, the fitting degree of equation 2 is $\mathrm{R}^{2}=0.850$ (reduced a little); upon adjustment, $\mathrm{R}^{2}=0.767$ (increased a little) and $\mathrm{F}=10.207$ (increased a little, $\mathrm{P}=0.002$ reveals improvement in significance level); the average annual standardized error of the equation is 0.297 (reduced a little); the standardized fitting error is 0.075 (reduced a little).

In the equation 2, three consumption items and two investment items produce positive effects, which reveal consumption contribution to GDP being greater than that of investment; and export impact on GDP being explained by the positive correlation between export $E_{4}$ and consumption $C_{2}$, investment $I_{1}$.

- Impact of important factors on No. 2 public factor of GDP

Export $E_{1}$ and GDP's No. 2 public factor $G_{2}$ are adopted to establish the regression equation, attaining a favorable effect. The standardized regression formula is as follows:

$$
G_{2}=-1.279 e^{-6}+0.918 E_{1}
$$

The fitting degree of equation 3 is $\mathrm{R} 2=0.831, \mathrm{~F}=69.61$ with significance level being outstanding $(\mathrm{P}=0.000)$. Obviously, export E1 has a high explained degree to G2.
Dependent Variable: gdp1

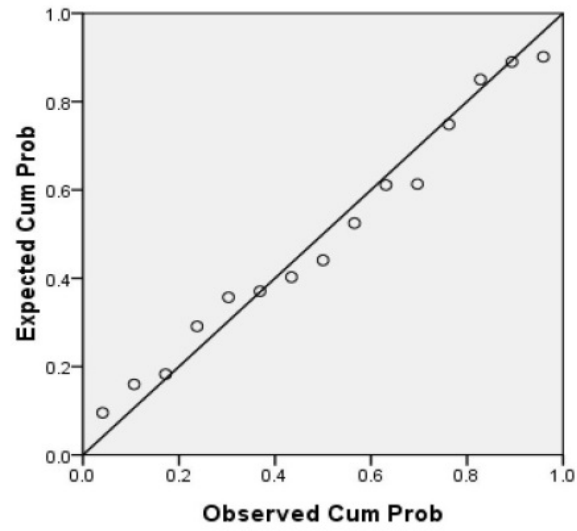

Normal P-P Plot of Regression Standardized Residual

Fig. 1. Distribution of accumulative frequency (showing residual) of the standardized regression for G1 equation 2

\section{Analysis of impact mechanism}

The 7 public factors $\left(I_{1}, I_{3}, C 1, C_{2}, C_{3}, E_{1}\right.$ and $\left.E_{4}\right)$ among regression equation 1 and equation 3 are selected as the Fig. 1 indicator to make hierarchical clustering analysis aiming at the sample cases over the years. For the sake of simple and clear analysis, the result divided to 4 year types of sample cases is taken to obtain 4 typical corresponding relationships (Table 2) between GDP growth and investment, consumption, export. We can conclude from the table that the total difference of economic indicators among 4 year types is evident(13.4\% 30.0\%) - Therefore, impact mechanism of the three economic factors on GDP growth showed in table 2 is worth paying attention to; it is visually expressed by Fig. 2a.

We can conclude from Table 2 and Fig.2a that GDP growth rate is high when investment and consumption are high despite export being medium high (it reflects the importance of investment and consumption on GDP's high-speed growth rate); when the consumption is low and investment is medium-low, GDP growth rate is low even though export is high (it reflects greater consumption impact and less export impact); when investment and consumption 
are medium-high and export is low, GDP is reduced to the growth rate of medium-low speed (export role can't be neglected); the impact of investment or consumption on GDP is obviously greater than that of export.

In order to demonstrate in a clearer manner, sequence is arranged year by year according to the average GDP growth rates of 31 provinces, and 4 year types with GDP growth rate

\section{E. Graded evaluation of present GDP growth potential}

gap are classified on basis of basically same year number. The results directly reflect some corresponding relationships between GDP growth rates and 3 economic indicators (Fig. 2b); this figure is only able to roughly demonstrate the common impact of 3 economic factors on GDP. We can conclude by comparing Fig.2a with Fig.2b that Fig.2a can reflect the impact of different combination of 3 factors on GDP growth rates and Fig.2b can’t do it.

TABLE II. ESSENTIAL CORRESPONDING RELATIONS BETWEEN THE GROWTH RATES OF 3 IMPORTANT ECONOMIC FACTORS AND GDP GROWTH LEVELS

\begin{tabular}{|c|c|c|c|c|c|c|}
\hline Year types & $\begin{array}{c}\text { Investment } \\
\text { (\%) }\end{array}$ & $\begin{array}{c}\text { Consumption } \\
\text { (\%) }\end{array}$ & $\begin{array}{l}\text { Export } \\
(\%)\end{array}$ & $\begin{array}{l}\text { GDP } \\
(\%)\end{array}$ & $\begin{array}{l}\text { Grades for growth rate of } \\
\text { GDP }\end{array}$ & Year case \\
\hline FL1 & 20.36 & 9.77 & 32.93 & 12.99 & Low & $2000 / 2003$ \\
\hline FL2 & 21.97 & 15.23 & 16.90 & 14.06 & Middle-low & 2001/2009/2010/ \\
\hline & & & & & & 2011/2012/2013 \\
\hline FL3 & 18.76 & 14.16 & 19.96 & 14.47 & High-middle & 1999/2002/2004/ \\
\hline & & & & & & 2008 \\
\hline FL4 & 25.56 & 16.31 & 27.89 & 18.66 & High & $2005 / 2006 / 2007$ \\
\hline Mean & 21.66 & 13.87 & 24.42 & 15.05 & & \\
\hline Standard deviation & 2.91 & 2.87 & 7.32 & 2.49 & & \\
\hline
\end{tabular}

Remark: This table refers to the sample clustering results based on 7 public factors from economic data issued by provincial statistic bureaus in China.

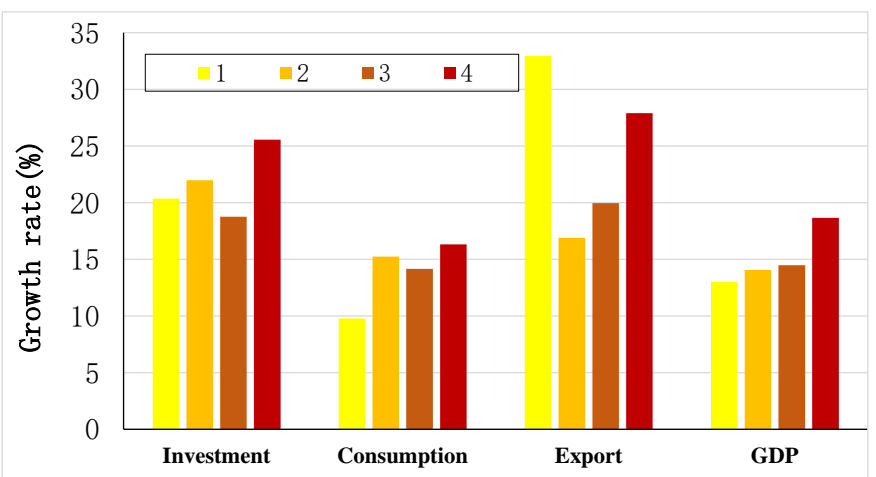

(a) the results of hierarchical clustering based on 7 public factors

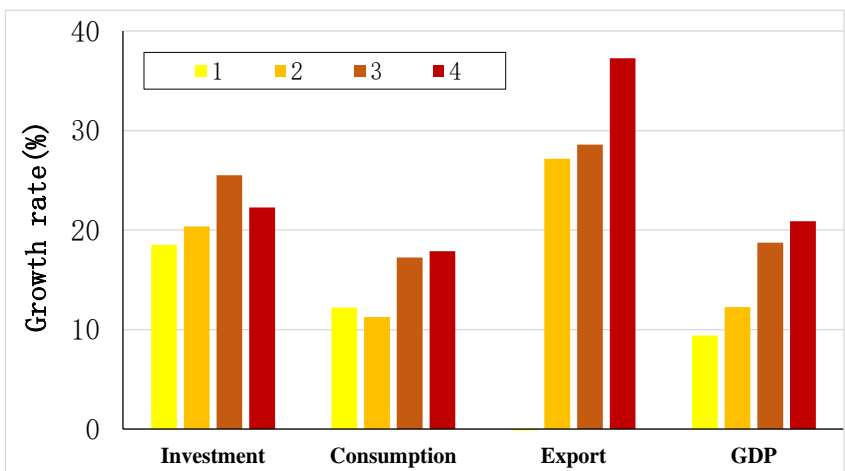

(b) sorting GDP growth grades based on original data

Fig. 2. Corresponding relationships between China’s GDP growth rates and the indexes of investment, consumption, export(Based on the economic data released by provincial statistic bureaus in China; a: essential relationship; b: superficial relationship)

The pearson correlation coefficient is adopted as a similarity index used to analyze the similarity between typical year types with different configurations of 3 economic indicators (Table 2) and the year 2013 by using the related data for various provinces. A bigger correlation coefficient means better similarity in the configuration of 3 important economic factors.

GDP growth potential level is evaluated and judged according to the following two bases: (1) the similarity of the size configuration for investment, consumption and export compared with the above-mentioned year types; (2) the average value of 3 economic indicators (demonstrating the general level in pushing GDP growth).

The evaluating and judging methods are determined as follows: firstly making use of the typical configuration of 4 year types and judging the most similar year type corresponded to the year 2013; then comparing the average value of 3 economic indicators in 2013 with the mean of the most similar year type; if the average value of 2013 is much different from the one of the most similar year type, a decreased or increased growth-rate grade by 1-2 levels compared the most similar year type is determined as the evaluated result.

Based on the above-determined touchstone and the related data in 2013, the evaluated results is obtained as follows: (1) 6 provinces (regions) including Chongqing, Gansu, Guizhou, Yunnan, Guangxi and He'nan are still in a middle-high growth rate stage of GDP; (2) 7 provinces (regions) including Hubei, Hunan, Hainan, Shanxi, Qinghai, Xinjiang and Ningxia are in middle-low growth rate stage of GDP; (3) The rest 18 provinces (regions) including 5 provinces of largest 
GDP ratio such as Beijing, Shanghai, Guangdong, Jiangsu and Zhejiang have entered into low growth rate stage. And no provinces or regions is still in high growth rate stage.

\section{DISCUSSION}

The investment is able to expand production and increase employment (favorable for consumption) while consumption and export are able to promote production and mobilize economic development, which promotes benign circulation[4]. The regression analysis and clustering analysis reveal that consumption and investment constitute the main and direct impact factors of GDP growth $[5,7,8]$. The positive effect of export on GDP growth is relatively less but can't be neglected.

According to the analysis of regression equation 1 and equation 2, with comprehensive considerations of the positive contribution rate (83.1\%) of export to G2 among regression formula (3); the export E4 being correlated positively to consumption C2 and investment I1 and negatively to export E1 and investment I3; and so on, the consumption contribution to GDP is greater than the positive effect of investment; the positive contribution rate of export is smaller than one of the previous two but still takes up a certain share.

According to the evaluation of 2013, 58\% provinces (regions) in the country (including top five economic provinces) have entered into low growth stage of GDP; $22.6 \%$ provinces have done middle-low growth stage of GDP. These data were supported by the fact released by China State Statistical Bureau: the annual growth rates of GDP were 7.62\%-9.08\% during 1998-2002; 2003-2010 was a high-speed growth stage of GDP (9.21\%-14.16\%); but growth rates were gradually reduced from $9.3 \%$ to $6.9 \%$ during 2011-2015 [9].

The source and difference of economic statistic data do not affect the objectiveness of analyzed results by this paper. China's statistical departments adopted and gradually promoted international economic investigation and statistic methods since the middle 1990s; however, the provincial statistical departments have certain differences with their State Statistical Bureau in using statistical calibers. Therefore, the corresponding values of four economic indicators for gained 4 GDP year types specified in Table 2 shows a larger order of magnitude (compared with the national annual GDP value released by Chinese government). In spite of these, the GDP values of various provinces (regions) are in accordance with the variation trend of national GDP issued by Chinese government. In another hand, several economic indicator fields all have certain public factors which are not correlated with the main public factors of GDP field- It may be caused by the source and deviation of statistical data.

\section{CONCLUSION}

The influence degree of three power-source factors on China GDP growth during 1998-2013 was made clear in this paper. The main-minor impact factors of Chinese GDP growth in order were determined as follow: consumption (C), investment (I), export (E) and import (Im). E was an important factor for GDP, but Im wasn't.

The typical graded quantitative relations between GDP growth and the index configuration for consumption, investment and export were found out. Based on the relations and the index mean values of above-mentioned three factors, a touchstone assessing the potential economic growth for an administrative region was put forward. Furthermore, according to the touchstone and the related economic data of 2013, it was determined that present Chinese economy had entered a low-speed growth period as a whole.

Consumption was the most important power-source factor for continuous and stable development of Chinese economy from 1998 to now. Increasing the power of consumption while maintaining a higher investment scale was a significant guarantee of maintaining sustainable and stable development of Chinese economy in near future. And promoting consumption and boosting domestic demands should be a key emphasis to Chinese government in adjusting economic structures during a new period.

The proper use of analysis methods was the key for getting results by this paper. The method of factor analysis played an important role in simplifying problems and stressing the key points. Correlation analysis and multiple regression jointly made clear the influence degree of four economic factors on Chinese GDP growth. Hierarchical clustering method found out the relationships between typical configurations of three power-source economic factors and the growth rates of Chinese GDP.

\section{REFERENCES}

[1] WU Jing-lian, "The realization of desirable 'New Normal' depends on reform,” in New Finance, vol.1, 2015, pp.7-10. (in Chinese with English abstract)

[2] LIU Qing-bao, WEI Liang-li, “An empirical study on the pulling function to Chinese economic growth from three power-source economic factors," in Special Zone Economy, vol.12, 2007, pp.257-258. (in Chinese with English abstract)

[3] SHEN Cheng-xiang, "An empirical analysis on the export-oriented economic growth in Chinese from 1977-1998,” in The Journal of World Economy, vol.12, 1999, pp.26-30. (in Chinese with English abstract)

[4] SHEN Li-sheng, "An assess on the pulling function of three power-source economic factors," in The Journal of Quantitative \& Technical Economics, vol.4, 2009, pp.139-151. (in Chinese with English abstract)

[5] ZHANG Li, "Consumption and investment: the motive power of rapid growth for Chinese economy,” in Statistics \& Decision, vol.1, 2003, pp.60-70. (in Chinese with English abstract)

[6] ZHANG Wen-tong, Tutorial of statistical analysis for SPSS11, Beijing: Hope electronic publishing house, 2002. (in Chinese)

[7] LONG Xia, “An empirical analysis on the relationship between economic growth and investment of permanent assets in China,” A master thesis of international finance from East China Normal University, 2006. (in Chinese with English abstract)

[8] RAO Xiao-hui, "The actual economic fluctuation and the productive outlays of government in China,” Economic Research Journal, vol.11, 2014, pp17-30. (in Chinese with English abstract)

[9] Data center of China State Statistics Bureau. Chinese GDP growth rates over the years. CHINA ECONOMIC NET, 2014-03-14, http://intl.ce.cn/zhuanti/data/s/Chinadata/201403/14/t20140314_248538 7.shtml (in Chinese) 